\title{
Pulsed Electric Fields (PEF) as pre-treatment for freeze-drying of plant tissues
}

\author{
Al-Sayed, L. a ; Boy, V. b*; Madieta, E. c; Mehinagic, E. c; Lanoisellé, J.-L. b \\ a Societal Transition and Agriculture (430 b), Institute of Social Sciences in Agriculture, University of \\ Hohenheim, 70599 Stuttgart, Germany \\ b Univ. Bretagne Sud, UMR CNRS 6027, IRDL, F-56300 Pontivy, France \\ c Unité GRAPPE, Ecole Superieure d'Agricultures Angers Loire (ESA), INRA, 55, rue Rabelais BP \\ 30748, 49007 Angers Cedex 01, France
}

*E-mail of the corresponding author: virginie.boy@univ-ubs.fr

\begin{abstract}
The influence of pulsed electric fields (PEF) treatment on freeze-drying for potato and strawberry tissues was investigated. Samples were pre-treated by PEF $\left(E=400 \mathrm{~V} \mathrm{~cm}^{-1}\right)$ for different treatment times. Freeze-drying was carried out at $-17^{\circ} \mathrm{C}$ and $18.4 \mathrm{~Pa}$ or $30 \mathrm{~Pa}$ for potato and strawberry tissues, respectively. The effects of PEF pre-treatment was compared with intact samples. The drying time was reduced by 35\% for potato and 30\% for strawberry. The sample rehydration capacity and the electrolytes released during the rehydration were higher for pre-treated samples. Strawberries texture was characterized by the hardness, the cohesiveness and the springiness.
\end{abstract}

Keywords: Pulsed Electric fields; Freeze-drying; Potato; Strawberry; Textural Properties. 


\section{Introduction}

Freeze-drying process is well-known to produce high quality products. However, the use of this process is limited because of the long drying time and the high energy consumption. For this reason, scientists keep searching for new pre-treatments to improve heat and mass transfers during the freeze-drying process without affecting the quality of the final products.

The relevance of using the pulsed electric fields (PEF) to enhance the performances for various modes of drying was pointed out. ${ }^{[1]}$ It was reported that, exposing a biological tissue to an external electric field for few microseconds cause a transcient increase in the transmembrane potential. This alters the organization of the cytoplasmic membrane by forming pores which increases the cell membrane permeabilization. ${ }^{[2]}$

A few studies have shown the ability of the PEF treatment on fruits and vegetables to accelerate the freeze-drying process by increasing the drying rate and/or reducing the drying time..$^{[3-6]}$

While these results are promising, researches are still needed to confirm these findings for different plant tissues and to examine the efficiency of the PEF assisted freeze-drying process on the quality of the final products. The objective of this work was to investigate the influence of coupling PEF treatment and freeze-drying on the dehydration kinetics of potato and strawberry tissues, and to investigate the effect of using this innovative process on the rehydration and textural properties of freeze-dried products.

\section{Materials and Methods}

\subsection{Sample preparation}

Potatoes (Agata) and strawberries (Charlotte and unknown variety from Spain) were bought from a local market (Pontivy, France) and stored in a refrigerator $\left(4^{\circ} \mathrm{C}\right)$. Initial moisture contents were $(85.2 \pm 1.0) \%$ and $(91.7 \pm 0.4) \%$ in wet basis $(w / w)$, respectively. Cylindrical potato $(18 \mathrm{~mm}$ diameter and $25 \mathrm{~mm}$ height) and strawberry samples (20 mm diameter and $27 \mathrm{~mm}$ height) were prepared.

\subsection{Pulsed Electric Field (PEF) pre-treatment}

The electric field treatment was carried out using a PEF generator $(2.5 \mathrm{kV} / 24 \mathrm{~mA}$, Effitech, Pau, France). The generator supplies near-rectangular monopolar pulses. $N$ series of pulses (-) were imposed during the treatment. Each series was composed of $n$ pulses (-) with pulse duration $t_{i}(\mu \mathrm{s})$ and pulse repetition time $\Delta t(\mathrm{~s})$. Two consecutive series were separated by an intertrain time $\Delta t_{t}(\min )$. This time between series was fixed at $5 \mathrm{~min}$ in 
order to control the increase in the product temperature $\left(10^{\circ} \mathrm{C}\right.$ in our case). The total PEF treatment time (s) was defined as $t_{t}=n \cdot N \cdot t_{i}$.

For potato samples, the PEF treatement was realized according to the following protocol: $E=400 \mathrm{~V} \mathrm{~cm}^{-1}, N=12.5, n=240, t_{i}=100 \mu \mathrm{s}, t_{t}=0.3 \mathrm{~s}$.

For strawberry samples, the same electric field strength, pulse duration and pulse number in each series were applied ( $E=400 \mathrm{~V} \mathrm{~cm}^{-1}, n=240, t_{i}=100 \mu \mathrm{s}$ ). For the study of the disintegration kinetics during the PEF treatment, $N=16$ series were required to reach the totally disintegrated tissue which corresponds to a PEF treatment time $t_{t}=0.384 \mathrm{~s}$. For the studies of the dehydration kinetics and the effect of PEF treatement on the freeze-dried strawberries, different number of series were applied $(N=0.5,1,2,3,4$ series) correspondent to various PEF treatment times $\left(t_{t}=0.012 \mathrm{~s}, 0.024 \mathrm{~s}, 0.036 \mathrm{~s}, 0.048 \mathrm{~s}\right.$ and $0.096 \mathrm{~s})$. After the PEF pre-treatment, the samples were frozen and stored in a freezer (Fryka Kältetechnic, Esslingen am Neckar, Germany) at $-80^{\circ} \mathrm{C}$ until needed.

The degree of tissues damage was assessed from the conductivity disintegration index $Z$ (-), calculated as $Z=\sigma-\sigma_{i} / \sigma_{d}-\sigma_{i} \cdot{ }^{[7]} \sigma$ is the electrical conductivity measured at time $t$ $\left(\mathrm{S} \mathrm{cm}^{-1}\right), \sigma_{i}$ the conductivity of the intact sample $\left(\mathrm{S} \mathrm{cm}^{-1}\right)$ and $\sigma_{d}$ the conductivity of the totally damaged sample $\left(\mathrm{S} \mathrm{cm}^{-1}\right) . \sigma_{d}$ was determined from the measurements of the electrical conductivity of a tissue after a freeze-thaw cycle.

\subsection{Freeze-drying experiments}

The freeze-drying experiments were carried out using a VirTis Advantage XL-70 freezedryer (Sp Scientific, Warminster, USA). The frozen samples $\left(-80^{\circ} \mathrm{C}\right)$ were introduced in the freeze-dryer on a pre-cooled heating shelf fixed at $-50^{\circ} \mathrm{C}$. For both products investigated, the temperature inside the freeze-dryer chamber was set at $-17^{\circ} \mathrm{C}$. The pressure inside the freeze-dryer chamber was kept at $18.4 \mathrm{~Pa}$ and $30 \mathrm{~Pa}$ for potato and strawberry tissues respectively. During the secondary drying, the plate temperature was risen to $45^{\circ} \mathrm{C}$. Triplicate samples were weighted using a precision balance (Acculab ALC-110.4, accuracy $10^{-4} \mathrm{~g}$, Sartorius, Goettingen, Germany). The relative mass, $\left(\mathrm{m} / \mathrm{m}_{i}\right)$ was calculated by dividing $m$, which is the measured mass at time $t$ (g) by $m_{i}$, the initial mass (g). 


\subsection{Quality analysis}

\subsubsection{Rehydration ratio}

The rehydration ratio was determined by weighing a sample before and after soaking it in distilled water at room temperature $(24 \pm 1)^{\circ} \mathrm{C}$ for one minute. These measurements were done for triplicate samples.

\subsubsection{Electrolyte release during rehydration}

The amount of electrolytes released during rehydration was determined by measuring the changes in electrical conductivity of the rehydration solution. These experiments were carried out with intact and PEF pre-treated samples with different treatment times $\left(t_{t}=0.012 \mathrm{~s}, 0.024 \mathrm{~s}, 0.048 \mathrm{~s}, 0.072 \mathrm{~s}\right.$ and $0.096 \mathrm{~s}$ ). The electric resistance of the solution was measured using an impedance meter (Agilent U1733C, Keysight Technologies, USA) at $100 \mathrm{KHz}$ frequency. The samples were first weighted and placed inside a small beaker filled with distilled water (conductivity of $0.001 \mathrm{~S} \mathrm{~m}^{-1}$ ). The liquid-solid ratio was fixed at 80. A magnetic stirrer was used to carefully agitate the mixture (100 $\operatorname{tr}^{-1 n^{-1}}$ ). A small quantity of the solution $(800 \mu \mathrm{L})$ was taken at different times during $2 \mathrm{~h}$ and was placed inside a cuvette electrodes (Eppendorf $800 \mu \mathrm{L}$ ), then it was connected to the impedance meter. The electrolyte release during rehydration was determined at room temperature $(24 \pm 1)^{\circ} \mathrm{C}$. These measurements were done for triplicate samples.

\subsubsection{Texture analysis}

The textural properties were determined through a Texture Analyser (MTS Synergie 200 H, Helmut Singer Elektronik, Aachen, Germany). Texture profile analysis TPA was carried out for freeze-dried slices of strawberry of $20 \mathrm{~mm}$ diameter. These textural measurements were performed with intact and PEF pre-treated samples with different treatment times (0.024 s, $0.048 \mathrm{~s}, 0.072 \mathrm{~s}$ and $0.096 \mathrm{~s})$. For each condition, twenty five replicates were analysed. The trials were done using a cylindrical probe of $25 \mathrm{~mm}$ diameter. Two compressions were realized with an interval of $5 \mathrm{~s}$ between cycles. The samples were compressed to $50 \%$ of their original height, using a load cell $1 \mathrm{kN}$ and the compression rate was $30 \mathrm{~mm} \mathrm{~min}^{-1}$. From the force-time curves, the hardness, the cohesiveness and the springiness were determined. ${ }^{[8]}$

\section{Results and discussion}

Fig. 1 presents the electrical conductivity disintegration index, $Z$, versus the total PEF treatment time for potato and strawberry tissues. 


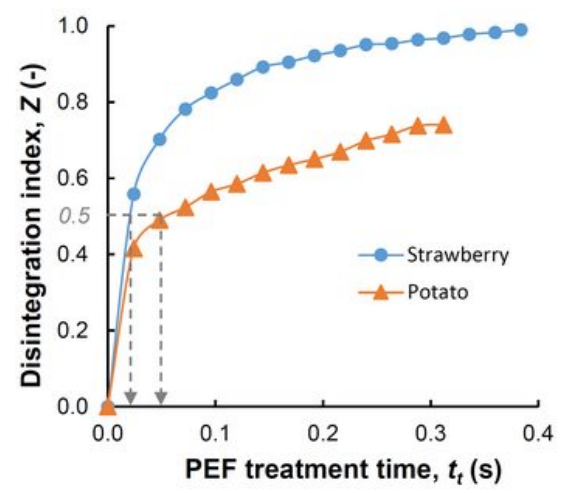

Fig. 1 Electrical conductivity disintegration index, $Z$, versus the total PEF treatment time for potato and strawberry tissues.

The disintegration index, $Z$, increases continuously during the PEF treatment. However, the evolution is not identical for potato and strawberry tissues. From Fig. 1, the halfpermeabilization, defined as the time for which the disintegration degree $Z=0.5$ is reached, was determined.

Strawberry is more sensitive to PEF treatment with a half-permeabilization happened after less than $0.024 \mathrm{~s}$ of treatment time compared to potato tissue which required a two-fold treatment time $(0.05 \mathrm{~s})$.

In order to investigate the benefits of PEF pre-treatement on the freeze-drying process, potato and strawberry were prepared, frozen at $-80^{\circ} \mathrm{C}$ and freeze-dried.

Fig. 2 depicts the relative mass of the sample, $\mathrm{m} / \mathrm{m}_{i}$, as a function of the freeze-drying time for strawberry samples.
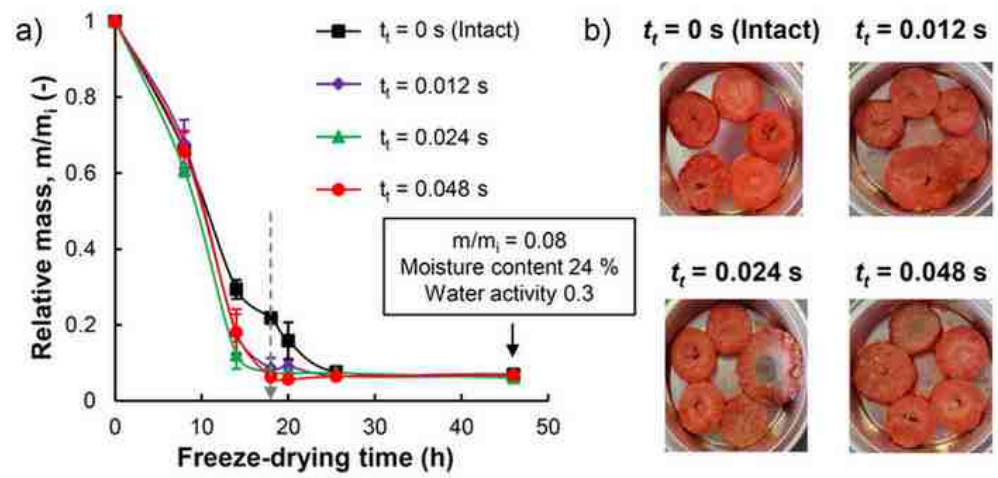

Fig. 2 a) Relative mass of the sample, $\mathrm{m} / \mathrm{m}_{i}$, as a function of the freeze-drying time for intact and PEF pre-treated strawberry samples with different treatment times; b) Photographies of samples. 
PEF pre-treatment accelerates the freeze-drying process and no difference is observable between the pre-treated samples with different treatment times (Fig. 2a). Eighteen hours are sufficient to dry the pre-treated samples, against $46 \mathrm{~h}$ for intact ones. Thus, the PEF pretreatment is able to reduce the drying time of strawberry by $30 \%$. Visually, the appearance of PEF pre-treated samples after freeze-drying is slightly damaged compared with intact ones (Fig. 2b). For potato samples, the positive effect of the PEF pre-treatment on the freeze-drying rate is also obtained and the drying time is shortenend by $35 \%$ (not shown).

The rehydration ratio is almost doubled for PEF pre-treated freeze-dried strawberries compared with intact ones. Moreover, the electrolyte release during the rehydration process increases with the PEF treatment time (Fig. 3).

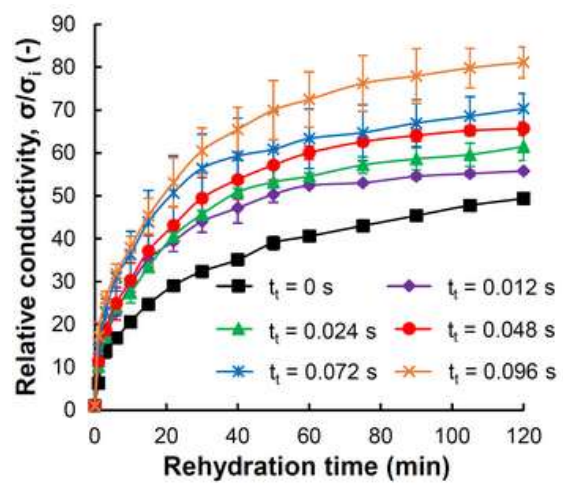

Fig. 3 Relative conductivity, $\sigma / \sigma_{i}$ as a function of time during the rehydratation of freeze-dried strawberries at different PEF treatment times.

These results come from the fact that PEF pre-treatment causes breaks in the cell membrane which facilitates the release of the cell components towards the extracellular medium. ${ }^{[9,3]}$ The concentration of the ionic components increases with the PEF treatment time as a consequence of a greater damage in the cell membrane. The statistical analysis indicated that PEF pre-treatment has a significant effect on the rehydration ratio (p-value $<0.05$ ).

Fig. 4 shows the average values of the hardness and the springiness for intact and pretreated freeze-dried strawberry slices for different PEF treatement times. 

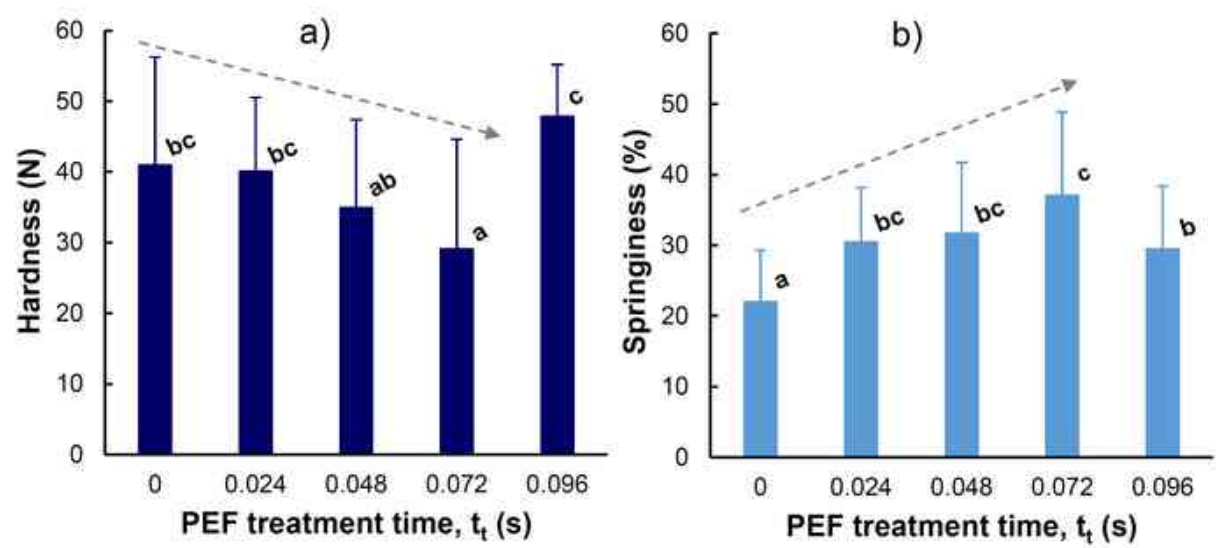

Fig. 4 Hardness and springiness for intact and pre-treated freeze-dried strawberry slices for different PEF treatment times.

The hardness represents the maximal force required to deform the sample in mastication during the first compression and the springiness characterizes the product elasticity. After the PEF pre-treatment, the strawberry tissues have lost a part of their initial strength. Thus, the hardness values decrease for increasing PEF treatment times until the time reaches $0.096 \mathrm{~s}$ (Fig. 4a). Both springiness and cohesiveness of pre-treated samples increase compared with the intact ones and this increase becomes all the more important as the PEF treatment time is high (springiness: Fig. $4 \mathrm{~b}$ and cohesiveness: not shown). All these results can be explained by the irreversible damage in the cell membrane caused by the PEF pretreatment. ${ }^{[10]}$ However, after an intensive PEF pre-treatement (0.096 s) the cells have lost their cellular turgor components and we assume that all the residues from the cells aggregate and forme a tougher structure.

\section{Conclusions}

The influence of coupling PEF treatment and freeze-drying process for potato and strawberry tissues was investigated. PEF pre-treatment was able to reduce the drying time of 35\% and 30\% for potato and strawberry, respectively. A significant rehydration capacity was found for pre-treated and freeze-dried strawberries. Strawberries texture was characterized by the hardness, the cohesiveness and the springiness.

\section{Acknowledgements}

Our gratitude goes to E. Mehinagic and E. Madieta who supervised the texture analysis. 


\section{References}

[1] Barba, F.J.; Parniakov, O.; Pereira, S.A.; Wiktor, A.; Grimi, N.; Boussetta, N.; Saraiva, J.A.; Raso, J.; Martin-Belloso, O.; Witrawa-Rajchert, D.; Lebovka, N.; Vorobiev, E. Current applications and new opportunities for the use of pulsed electric fields in food science and industry. Food Research International 2015, 77 (4), 773-798.

[2] Vorobiev, E.; Lebovka, N. Electrotechnologies for extraction from food plants and biomaterials; Springer: New York, 2008.

[3] Jalté, M.; Lanoisellé, J.-L.; Lebovka, N.I. ; Vorobiev, E. Freezing of potato tissue pretreated by pulsed electric fields. LWT - Food Science and Technology 2009, 42 (2), 576-580.

[4] Ben Ammar, J.; Lanoisellé, J.-L. ; Lebovka, N.I. ; Van Hecke, E. ; Vorobiev, E. Effect of a pulsed electric field and osmotic treatment on freezing of potato tissue. Food Biophysics 2010, 5 (3), 247-254.

[5] Wu, Y.; Guo, Y.; Zhang, D. Study of the effect of high-pulsed electric field treatment on vaccum freeze-drying of apples. Drying Technology 2011, 29 (14), 1714-1720.

[6] Parniakov, O.; Bals, O.; Lebovka, N.; Vorobiev, E. Pulsed electric fields assisted vacuum freeze-drying of apple tissue. Innovative Food Science and Emerging Technologies 2016, 35, 52-57.

[7] Lebovka, N.I.; Bazhal, M.I.; Vorobiev, E. Estimation of characteristic damage time of food materials in pulsed-electric fields. Journal of Food Engineering 2002, 54 (4), 337 346.

[8] Szczesniak, A. S. Texture is a sensory property. Food Quality and Preference 2002, 13 (4), 215-225.

[9] Taiwo, K.A.; Angersbach, A.; Knorr, D. Influence of high intensity electric field pulses and osmotic dehydration on the rehydration characteristics of apple slices at different temperatures. Journal of Food Engineering 2002, 52 (2), 185-192.

[10] Lebovka, N.I.; Praporscic, I.; Vorobiev, E. Effect of moderate thermal and pulsed electric field treatments on textural properties of carrots, potatoes and apples. Innovative Food Science \& Emerging Technologies 2004, 5 (1), 9-16. 